\title{
Momentum Theorems and the Structure of Atmospheric Jets and Zonal Flows in Plasmas
}

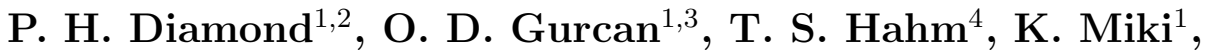 \\ Y. Kosuga ${ }^{1}$ and X. Garbet ${ }^{3}$ \\ ${ }^{1}$ Center for Astrophysics and Space Science, U.C.S.D., La Jolla, CA 92093, USA. \\ ${ }^{2}$ Kavli Institute for Theoretical Physics, U.C.S.B., Santa Barbara, CA 93106, USA. \\ 3 Association Euratom-C.E.A., CEA/DSM/IRFM, CEA-Cadarache, F-13108 St. \\ Paul-lez-Durance Cedex, France. \\ ${ }^{4}$ Princeton University, Princeton Plasma Physics Laboratory, P.O. Box 451, \\ Princeton, New Jersey 08543, USA. \\ PACS numbers: 52.30.Ex, 52.35.Ra, 52.35.We
}

\begin{abstract}
The inviscid invariance of potential vorticity is used to derive momentum balance relations for zonal flows in drift wave turbulence. The relations are constructed by exploiting potential enstrophy balance and the Taylor identity, and link flow momentum to turbulence pseudomomentum, along with the driving flux, the dissipation and turbulence spreading. Applications to atmospheric jets and to zonal flows in plasmas are discussed.
\end{abstract}

Keywords: turbulence, plasmas, waves

\section{Introduction}

Zonal flow physics has been the focus of a very large research effect in the magnetic confinement physics community[1, 2]. However, while its certainly the case that considerable progress has been achieved, many aspects of zonal flow dynamics remain poorly understood. In this paper, we present a brief summary of ongoing research on momentum theorems for zonal flows in quasi-geostrophic and drift wave turbulence. In contrast to most, if not all, of the previous theoretical studies of zonal flow dynamics in the M.F.E. community, here we concentrate on a.) zonal flow momentum, rather than energy, and its relation to fluxes, entrainment, dissipation, etc., b.) the spatial structure of zonal flows, rather than the dynamics of $\mathbf{k}$-space nonlinear couplings which excite them. We forego the usual picture, couched in terms of microscopics and modulational instability, spectral transfer, etc., in favor of one linked to macroscopics and spatial transport, c.) the impact of entrainment effects, aka 'turbulence spreading', on flow dynamics. We give special attention to the effect of the potential enstrophy flux on zonal flow evolution, and argue that turbulence spreading can actually drive shear flows.

Our discussion, which is partly tutorial and partly original, is centered on two case studies, which are: 
i.) $\beta$-plane models of the generation of mid-latitude westward jets in Rossby wave turbulence[3],

ii.) the structure of zonal flows in Hasegawa-Wakatani[4] models of drift wave turbulence with fixed driving flux.

The principal results of this analysis are zonal momentum balance theorems, akin to those of Charney and Drazin[5], which relate the evolution of zonal flow momentum to that of the turbulence pseudomomentum[6] and physical processes controlling relaxation and dissipation in drift wave turbulence. The pseudomomentum (sometimes referred to as the wave activity density[7]) may be thought of as a wave or turbulence momentum density, is proportional to the familiar wave action density in the weakly nonlinear limit, and is unambiguously defined via the evolution of potential enstrophy density (locally conserved for inviscid dynamics) and the Taylor identity between vorticity flux and Reynolds stress. Unlike wave kinetics, however, this formulation is not restricted to regimes of weak turbulence. The utility of potential enstrophy balance and the Taylor identity are unifying themes of this paper.

\section{Jet Formation in $\beta$-plane Turbulence}

A well known and instructive case-study in the formation of zonal flows is that of the formation of mid-latitude westward jets. This zonal jet is part of a ubiquitous dipole pair consisting of an eastward subtropical jet and a westward jet at mid-latitudes, which necessarily flows against the prevailing atmospheric wind. Turbulence is excited on the subtropics due to baroclinic instability, etc. Moreover, the excitation inputs no net momentum, so the zonal flow direction must be set by the system properties (i.e. coriolis force, wave propagation, direction, etc.) alone. This suggests that the problem

of dipole formation reduces to one of understanding how adding energy at low latitudes accelerates the eastward flow in the subtropics while simultaneously driving a westward jet at mid-latitudes.

The zonally averaged flow $\left\langle v_{x}\right\rangle$ evolves according to,

$$
\frac{\partial\left\langle v_{x}\right\rangle}{\partial t}=-\frac{\partial}{\partial y}\left\langle\tilde{v}_{y} \tilde{v}_{x}\right\rangle-\nu\left\langle v_{x}\right\rangle
$$

where we have included a scale independent friction $\nu$ to allow stationarity of the mean zonal flow. For GFD applications, this friction may be thought of as corresponding to topographic friction, while for plasma applications, $\nu$ corresponds to collisional drag.

The Taylor identity for geostrophic flow,

$$
-\frac{\partial}{\partial y}\left\langle\tilde{v}_{y} \tilde{v}_{x}\right\rangle=\left\langle\tilde{v}_{y} \tilde{\omega}\right\rangle
$$

in turn relates the Reynolds force to the flux of vorticity in latitude. The plasma analogue of this useful relation is an identity between the flux of polarization charge and the Reynolds force exerted by fluctuating $\mathbf{E} \times \mathbf{B}$ flows. As the flux of vorticity also appears in the production term of the enstrophy evolution equation, we 
straightforwardly derive the latter from the well-known quasi-geostrophic equation (a closely related antecedent of the familiar Hasegawa-Mima[8] equation),

$$
\begin{aligned}
& \frac{\partial}{\partial t} \tilde{\omega}+\tilde{\mathbf{v}} \cdot \nabla \tilde{\omega}=-\left(\beta+\frac{\partial}{\partial y}\langle\omega\rangle\right) \tilde{v}_{y}+\mu \nabla^{2} \tilde{\omega}+\tilde{f} \\
& \frac{\partial}{\partial t}\left\langle\tilde{\omega}^{2}\right\rangle+\frac{\partial}{\partial y}\left\langle\tilde{v}_{y} \tilde{\omega}^{2}\right\rangle+\beta^{*}\left\langle\tilde{v}_{y} \tilde{\omega}\right\rangle=\langle\tilde{f} \tilde{\omega}\rangle-\mu\left\langle(\nabla \tilde{\omega})^{2}\right\rangle .
\end{aligned}
$$

Here $\beta^{*} \equiv \beta+\partial\langle\omega\rangle / \partial y,\left\langle\tilde{\omega}^{2}\right\rangle$ is the zonally averaged enstrophy density, and a factor of 2 has been absorbed into $\beta^{*}, \tilde{f}$ and $\mu$. Equation $(2 b)$ states that enstrophy density is produced by stirring and by interaction with $\beta^{*}$, damped by viscosity, and transported by advection. Combining Eqns. (1a, 1b, 2b) yields:

$$
\frac{\partial}{\partial t}\left[\frac{\left\langle\tilde{\omega}^{2}\right\rangle}{\beta^{*}}+\left\langle v_{x}\right\rangle\right]=\frac{\langle\tilde{f} \tilde{\omega}\rangle}{\beta^{*}}-\frac{\mu}{\beta^{*}}\left\langle(\nabla \tilde{\omega})^{2}\right\rangle-\frac{1}{\beta^{*}} \frac{\partial}{\partial y}\left\langle\tilde{v}_{y} \tilde{\omega}^{2}\right\rangle-\nu\left\langle v_{x}\right\rangle,
$$

which is a momentum conservation theorem for the zonal flow $\left\langle v_{x}\right\rangle$. The quantity $\left\langle\tilde{\omega}^{2}\right\rangle / \beta^{*}$ may be recognized as the negative of the turbulence pseudomomentum, which for small amplitudes is equivalent to the zonal momentum density of Rossby waves $k_{x} N(\mathbf{x}, \mathbf{k}, t)$, where $N$ is the wave action density. Thus, Eqn. (3) states that (to within a constant), the zonal flow momentum is locked to the wave momentum density, apart from the effects of forcing, dissipation and the spatial flux of wave momentum density. Equivalently, Eqn. (3) also implies that in the absence of forcing, dissipation and wave momentum density flux, stationary turbulence cannot accelerate a mean zonal flow. Note that Eqn. (3) is in principle exact, and makes no approximation of small amplitude, weakly nonlinear waves, quasilinear closure, etc. Thus, while the wave activity density $\left\langle\tilde{\omega}^{2}\right\rangle / \beta^{*}$ is easily seen to be related to the wave momentum density in cases of 'weak' or 'wave' turbulence, it is in fact much more general. Reduction to weak or wave turbulence requires that the enstrophy density flux term $\left(1 / \beta^{*}\right)(\partial / \partial y)\left\langle\tilde{v}_{y} \tilde{\omega}^{2}\right\rangle$ be negligible, or at least smaller than the quadratic terms in Eqn. (3). In practice, this occurs when $\tilde{v}_{y} / L_{\mathrm{E}} \ll 1 / \tau$, i.e. when turbulent advection over the enstrophy density envelope scale $L_{\mathrm{E}}$ is slow in comparison to rates characteristic of enstrophy density evolution. Thus, reduction to the weak turbulence or quasi-linear limit occurs when $L_{\mathrm{E}}$ is sufficiently large, regardless of the turbulence intensity.

The momentum theorem of Eqn. (3) has interesting implications for the problem of mid-latitude westward jet formation. This follows from consideration of Eqn. (3) for stationary turbulence and flows, which may easily be solved for the zonal velocity $\left\langle\tilde{v}_{x}\right\rangle$, i.e.

$$
\left\langle v_{x}\right\rangle=\frac{1}{\nu \beta^{*}}\left[\langle\tilde{f} \tilde{\omega}\rangle-\mu\left\langle(\nabla \tilde{\omega})^{2}\right\rangle-\frac{\partial}{\partial y}\left\langle\tilde{v}_{y} \tilde{\omega}^{2}\right\rangle\right] .
$$

The limiting case where $\beta^{*} \rightarrow 0$, which corresponds to constant total mean potential vorticity, will be discussed in a future publication. Ignoring, for the moment, the enstrophy flux, Eqn. (4) relates the zonal velocity to forcing and viscous dissipation of enstrophy. In particular, for forcing $\tilde{f}$ sharply localized in the subtropics, $\left\langle v_{x}\right\rangle \cong$ $\langle\tilde{f} \tilde{\omega}\rangle / \nu \beta^{*} \approx\left\langle\tilde{f}^{2}\right\rangle \tau_{c} / \nu \beta^{*}$. Here $\tau_{c}$ is the correlation time for the response of $\tilde{\omega}$ to $\tilde{f}$. Note 
that the direction of $\left\langle v_{x}\right\rangle$ (i.e. eastward) is set by $\beta^{*}$, despite the absence of any net momentum input or net orientation in the external forcing. Away from the subtropics, where forcing is negligible, $\left\langle v_{x}\right\rangle \cong-\left(\mu / \nu \beta^{*}\right)\left\langle(\nabla \tilde{\omega})^{2}\right\rangle$, so the zonal flow is westward, consistent with the formation of mid-latitude westward jets. Thus, we see that a dipole pair of jets can results from the combined action of localized forcing and distributed dissipation. Finally, note that within the $\beta$-plane approximation, conservation of total zonal momentum is assured by conservation of total enstrophy, since

$$
\begin{aligned}
\int \mathrm{dy}\left\langle\mathrm{v}_{\mathrm{y}}\right\rangle & =\frac{1}{\nu \beta^{*}} \int \mathrm{dy}\left[\langle\tilde{\mathrm{f}} \tilde{\omega}\rangle-\mu\left\langle(\nabla \tilde{\omega})^{2}\right\rangle\right]-\left.\frac{1}{\nu \beta^{*}}\left\langle\tilde{\mathrm{v}}_{\mathrm{y}} \tilde{\omega}^{2}\right\rangle\right|_{y_{-}} ^{y_{+}} \\
& =0,
\end{aligned}
$$

i.e. in a stationary state, enstrophy input from forcing must equal losses due to enstrophy dissipation and enstrophy flux thru the boundary of the region of interest. Its interesting to observe that as a consequence of the Taylor identity, zonal flow momentum conservation is linked directly to enstrophy balance, and that zonal flow structure is determined by the profiles of stirring and dissipation.

The physical meaning of Eqn. (3) merits discussion, so here we offer a novel interpretation of this useful result. Given that $\left\langle\tilde{\omega}^{2}\right\rangle / \beta^{*}$ is the negative pseudomomentum, Eqn. (3), which evolves the difference of flow and wave momentum, may be thought of as a relation governing the relative slip, i.e. the difference between the mean momentum of the fluid $\left\langle v_{x}\right\rangle$ and the momentum of a co-existing, inter-penetrating fluid of quasiparticles (i.e. $-\left\langle\tilde{\omega}^{2}\right\rangle / \beta^{*}$ ) which are simply the waves, vortices etc. of the turbulence. In the absence of direct excitation or dissipation of the quasi-particles or fluid, Eqn. (3) states that these two fluids are locked together or frozen into one another, so that their relative momentum cannot change. Thus, in the absence of dissipation etc., and for stationary turbulence, the zonal flow cannot grow at the expense of the fluctuations since this would violate the 'no-slip' law. Indeed, zonal flow evolution requires some relative pumping of the fluctuations or fluctuation damping (i.e. a 'beach' effect) or a net spatial in/out flow of fluctuation pseudomomentum (i.e. $\sim \partial_{y}\left\langle\tilde{v}_{y} \tilde{\omega}^{2}\right\rangle$ ), all of which can potentially alter the balance between the momentum of the physical fluid and that of the quasi-particle fluid, thus allowing one to slip relative to the other. In this sense, the result of Eqn. (3) may be viewed as a consistency condition for coupling in the presence of dissipation, since it specifies precisely what flow is required for the drag on the physical fluid to balance the change in the momentum of the quasi-particle fluid. Future work on this topic will address the important issue of Galilean invariance.

An even simpler explanation of the dipole jet formation may be obtained from simple considerations of Rossby wave energy and momentum flux. Of course, the Rossby wave is the linear eigenmode of the quasi-geostrophic equation with dispersion relation,

$$
\omega=-\beta k_{x} / k^{2}+k_{x}\left\langle v_{x}\right\rangle,
$$

where we include the effect of a zonal flow in the $\hat{x}$ direction. Rossby waves, excited by energy input at the equator, must radiate energy poleward. Causality requires the consequent outgoing wave boundary condition to force perturbations to vanish as 
$y \rightarrow \infty$, so upon introducing an infinitesimal drag $\gamma, \delta k_{y}>0$, i.e. the resulting increment in $k_{y}$ must be positive. Since $\delta k_{y}=i \gamma /\left(\partial \omega_{r} / \partial k_{y}\right)$, this requires the product $k_{x} k_{y}>0$. However, the wave-induced momentum flux is just

$$
\Pi_{y, x}=\sum_{\mathbf{k}}-k_{y} k_{x}\left|\tilde{\phi}_{\mathbf{k}}\right|^{2}
$$

where $\phi$ is the stream function. Thus, $\Pi_{y, x}<0$, and so always transports momentum towards the equator. This guarantees that as energy radiates away from equatorial excitation, momentum will coverage there, thus accelerating an eastward equatorial jet. Since the equatorial convergence of momentum necessarily implies a momentum deficit at higher latitudes, a westward jet must necessarily form there. This accounts for the formation of dipole pairs of jets. Interestingly, since the Rossby wave group velocity $v_{g y}=2 \beta k_{x} k_{y} /\left(k_{x}^{2}+k_{y}^{2}\right)^{2}$ is positive definite (since $k_{x} k_{y}>0$ ), equatorial momentum convergence is a simple consequence of the fact that Rossby waves are 'backward', i.e. that their latitudinal phase and group velocities are always opposite, so $v_{p h y} v_{g y}<0$, thus reconciling a poleward energy flux with an equatorward momentum flux. Finally, it is only meaningful to impose outgoing wave boundary conditions when the excitation region is localized relative to the dissipation region.

\section{Zonal Flow Momentum in Drift Wave Turbulence}

We now consider zonal flow momentum in a simplest nontrivial model of drift wave turbulence, namely the Hasegawa-Wakatani(HW) model, contained in the density and vorticity equations:

$$
\begin{aligned}
& \frac{\mathrm{d} n}{\mathrm{~d} t}=-D_{\|} \nabla_{\|}^{2}(\phi-n)+D_{0} \nabla^{2} n, \\
& \frac{\mathrm{d}}{\mathrm{d} t} \nabla^{2} \phi=-D_{\|} \nabla_{\|}^{2}(\phi-n)+\mu \nabla^{2} \nabla^{2} \phi .
\end{aligned}
$$

Here $D_{\|}$refers to parallel collisional diffusion - i.e. $D_{\|}=v_{\text {The }}^{2} / \nu_{\text {ei }}$ - while $D_{0}$ and $\mu$ are the perpendicular collisional diffusivity and viscosity, respectively, and the rest of the notation is standard. Hereafter, we take Prandtl number $\operatorname{Pr}=\mu / D_{0}=1$, for simplicity. $\operatorname{Pr} \neq 1$ will be discussed in a future publication. The HW model is the 'minimally relevant' one since parallel dissipation shifts the phase between $\tilde{v}_{r}$ and $\tilde{n}$, allowing resistive drift wave instability, the self-excitation of turbulence, and a finite turbulent particle flux $\Gamma_{\mathrm{T}}=\left\langle\tilde{v}_{r} \tilde{n}\right\rangle \neq 0$. The evolution of the mean flow is identical to that given in Eqn. (1a).

A key lesson of Section 2 is the utility of the potential enstrophy budget in constraining the zonal flow evolution. Hence, it is interesting to note that the explicitly 3D HW system locally evolves the potential vorticity (PV),

$$
u=\nabla^{2} \phi-n \text {. }
$$

This is conserved since it obeys the intrinsically $2 D$ equation,

$$
\frac{\mathrm{d} u}{\mathrm{~d} t}=D_{0} \nabla^{2} u
$$


Of course, for Boltzmann electrons where $n=\phi$, Eqn. (9b) reduces to the familiar Hasegawa-Mima equation. Since PV is conserved along particle trajectories up to diffusion, potential enstrophy $\left\langle u^{2}\right\rangle$ is an inviscid invariant. Thus, a fluctuation potential enstrophy density balance relation of the form,

$$
\frac{\partial}{\partial t}\left\langle\tilde{u}^{2}\right\rangle+\frac{\partial}{\partial r}\left\langle\tilde{v}_{r} \tilde{u}^{2}\right\rangle=-\left\langle\tilde{v}_{r} \tilde{u}\right\rangle \frac{\mathrm{d}\langle u\rangle}{\mathrm{d} r}-D_{0}\left\langle(\nabla \tilde{u})^{2}\right\rangle
$$

is easily obtained. As before, the potential enstrophy budget balances production via PV flux $\left\langle\tilde{v}_{r} \tilde{u}\right\rangle$ (acting with $\mathrm{d}\langle\mathrm{u}\rangle / \mathrm{dr}$ ) with diffusive damping and advection of potential enstrophy density. Since the zonally averaged flow satisfies

$$
\frac{\partial}{\partial t}\left\langle v_{\theta}\right\rangle=\left\langle\tilde{v}_{r} \nabla^{2} \tilde{\phi}\right\rangle-\nu\left\langle v_{\theta}\right\rangle
$$

via the Taylor identity, we can straightforwardly obtain a zonal momentum conservation theorem of the form

$$
\begin{aligned}
\frac{\partial}{\partial t}\left\{\frac{\left\langle\tilde{u}^{2}\right\rangle}{\mathrm{d}\langle\mathrm{u}\rangle / \mathrm{dr}}+\left\langle v_{\theta}\right\rangle\right\} & =\left\langle\tilde{v}_{r} \tilde{n}\right\rangle \\
& -\left(\frac{\mathrm{d}\langle u\rangle}{\mathrm{d} r}\right)^{-1}\left\{D_{0}\left\langle(\nabla \tilde{u})^{2}\right\rangle+\partial_{r}\left\langle\tilde{v}_{r} \tilde{u}^{2}\right\rangle\right\}-\nu\left\langle v_{\theta}\right\rangle
\end{aligned}
$$

This theorem is the principal result of this paper. Equation (11) relates the sum of the drift wave activity density (the negative of the pseudomomentum) and the zonal flow momentum to the driving flux, the potential enstrophy dissipation, the potential enstrophy density flux, and the drag. Note that in the absence of turbulent transport, dissipation and entrainment, Eqn. (11) states that stationary turbulence cannot excite a zonal flow. Equation (11) establishes an intimate connection between particle flux and poloidal flow, since it shows that a spike in $\left\langle\tilde{v}_{r} \tilde{n}\right\rangle$ will necessarily excite a response in $\left\langle v_{\theta}\right\rangle[9]$. Moreover, the zonal flow momentum can be constrained further by noting that in nearly all applications to core transport, the total driving flux should be regarded as fixed, so

$$
\Gamma_{0}=\left\langle\tilde{v}_{r} \tilde{n}\right\rangle-D_{0} \frac{\partial\langle n\rangle}{\partial r}
$$

is a constant, and Eqn. (11) can be re-written as

$$
\begin{aligned}
\frac{\partial}{\partial t}\left\{\frac{\left\langle\tilde{u}^{2}\right\rangle}{\mathrm{d}\langle\mathrm{u}\rangle / \mathrm{dr}}+\left\langle v_{\theta}\right\rangle\right\} & =\Gamma_{0}+D_{0} \partial\langle n\rangle / \partial r \\
& -\left(\frac{\partial\langle u\rangle}{\partial r}\right)^{-1}\left\{D_{0}\left\langle(\nabla \tilde{u})^{2}\right\rangle+\partial_{r}\left\langle\tilde{v}_{r} \tilde{u}^{2}\right\rangle\right\}-\nu\left\langle v_{\theta}\right\rangle .(13)
\end{aligned}
$$

In general, the collisional particle transport, $D_{0} \partial\langle n\rangle / \partial r$ term is negligible in comparison to $\Gamma_{0}$, with the exception of regimes where the turbulent flux is quenched, such as in the case of transport barriers or the Dimits shift[10]. For stationary states corresponding to L-mode regimes, Eqn. (13) yields an explicit expression for the zonal flow in the form

$$
\left\langle v_{\theta}\right\rangle=\frac{1}{\nu}\left\{\Gamma_{0}-\frac{1}{(\partial\langle u\rangle / \partial r)}\left[D_{0}\left\langle(\nabla \tilde{u})^{2}\right\rangle+\partial_{r}\left\langle\tilde{v}_{r} \tilde{u}^{2}\right\rangle\right]\right\}
$$


Equation (14) is notable as it relates the saturated zonal flow to the driving fixed flux $\Gamma_{0}$, the viscous potential enstrophy density dissipation and the radial flux of potential enstrophy density. It departs from previous formulations in that the dependence on linear growth, etc. - ubiquitous in modulational stability calculations - is replaced by proportionality to the fixed constant driving flux $\Gamma_{0}$, which is a macroscopic quantity. Reynolds stress no longer appears explicitly, but is replaced by dependence on potential enstrophy dissipation and transport. Equation (14) again echoes the theme that zonal flow structure is regulated by dissipation profiles. In particular, it states that the all important zonal flow shear $\left\langle v_{\theta}\right\rangle^{\prime}$ is set by

$$
\left\langle v_{\theta}\right\rangle^{\prime}=-\frac{\Gamma_{0}}{\nu^{2}} \nu^{\prime}-\left\{\frac{1}{\nu\langle u\rangle^{\prime}}\left(D_{0}\left\langle(\nabla \tilde{u})^{2}\right\rangle+\partial_{r}\left\langle\tilde{v}_{r} \tilde{u}^{2}\right\rangle\right)\right\}^{\prime},
$$

i.e. by the radial profile of the drag and the potential enstrophy dissipation. This, in turn, suggests that drag and dissipation profiles, as well as turbulence spreading, are essential elements of the shear suppression feedback loop, and that quasi-local flux-tube models which ignore them make a serious omission. We also note that as $\left\langle v_{\theta}\right\rangle^{\prime}$ increases $\left\langle\tilde{v}_{r} \tilde{n}\right\rangle$ must decrease to the point that the collisional flux is no longer negligible. Hence, this contribution must be retained to accurately describe or simulate zonal flows at the threshold of transport barrier formation or in the Dimits shift.

An intriguing and novel feature of Eqns $(13,14)$ is the apparent impact of potential enstrophy density flux on zonal flow dynamics. Equation (13) implies that jumps in the flux $\left\langle\tilde{v}_{r} \tilde{u}^{2}\right\rangle$ can drive a zonal flow in the transition region, i.e. that a change in $\left\langle\tilde{v}_{r} \tilde{u}^{2}\right\rangle$ over a layer of width $\Delta r$ can drive a flow on that scale according to the approximate relation:

$$
\left.\left(\partial_{t}\left\langle v_{\theta}\right\rangle\right) \Delta r\left(\frac{\partial\langle u\rangle}{\partial r}\right) \approx\left\langle\tilde{v}_{r} \tilde{u}^{2}\right\rangle\right|_{x_{+}} ^{x_{-}} .
$$

This suggests that poloidal rotation associated with the onset of Internal Transport Barriers (ITB's) may arise from the drop in the turbulent potential enstrophy flux associated with barrier formation. It also states that a feedback loop involving turbulence spreading (of $\left\langle\tilde{u}^{2}\right\rangle$ ) can play a role in barrier formation via the scenario where: Note that this scenario should not be regarded as counter-intuitive, since Eqn. (13) states that zonal momentum evolution is strongly tied to pseudomomentum evolution. Since the pseudomomentum is in turn directly proportional to $\left\langle\tilde{u}^{2}\right\rangle$ (the potential enstrophy density), transport of $\left\langle\tilde{u}^{2}\right\rangle$ must indeed influence the evolution of zonal momentum. More generally, Eqn. (13) also suggests that transport of potential enstrophy can act as a mechanism of nonlinear saturation for zonal flows in the collisionless limit (i.e. when drag $\nu \rightarrow 0$ ). In that case, for stationary turbulence (and ignoring all collisional flow dissipation)

$$
\frac{\partial\left\langle v_{\theta}\right\rangle}{\partial t}=\left\langle\tilde{v}_{r} \tilde{n}\right\rangle-\frac{1}{\langle u\rangle^{\prime}} \frac{\partial}{\partial r}\left\langle\tilde{v}_{r} \tilde{u}^{2}\right\rangle
$$

so saturation is in principle possible if $\left\langle\tilde{v}_{r} \tilde{u}^{2}\right\rangle \sim \int \operatorname{dr}\left\langle\tilde{\mathrm{v}}_{\mathrm{r}} \tilde{\mathrm{n}}\right\rangle\langle\mathrm{u}\rangle^{\prime}$, i.e. if the potential enstrophy flux balances the net potential enstrophy production by the particle flux. 
Note that while we have known for sometime that turbulence spreading[11] may act as a saturation mechanism, this argument pinpoints the transport of potential enstrophy as that which critical to zonal flow dynamics. The theoretical technology to explicitly compute $\left\langle\tilde{v}_{r} \tilde{u}^{2}\right\rangle$ already exists in the literature[12]. Finally Eqns. (13,16) suggest that a flux of potential enstrophy thru the plasma boundary may induce a net poloidal rotation.

Another interesting feature of Eqn. (11) is the unambiguous emergence of $-\left\langle\tilde{u}^{2}\right\rangle /\langle u\rangle^{\prime}$ as the turbulence pseudomomentum or 'wave momentum density', i.e. the intensity - dependent quantity which is locked together with the zonal flow momentum. While $-\left\langle\tilde{u}^{2}\right\rangle /\langle u\rangle^{\prime}$ surely reduces to the familiar expression $k_{\theta} N$ (i.e. the poloidal momentum density of mode $\mathbf{k}$ ) in the limit of weak turbulence and Boltzmann electrons, it is far more general since it follows from enstrophy balance and the Taylor identity, alone, and is not based upon wave kinetics, which is sensitive to the structure of the linear dispersion relation. In particular, the structure of the conservation equation for the quantity $\left(\left\langle\tilde{u}^{2}\right\rangle /\langle u\rangle^{\prime}+\left\langle v_{\theta}\right\rangle\right)$ (i.e. zonal flow momentum - pseudomomentum), does not depend upon whether the turbulence is weak or strong, whether the electrons are Boltzmann (i.e. $D_{\|} /(R q)^{2} \omega_{*} \gg 1$ ) or hydrodynamic (i.e. $D_{\|} /(R q)^{2} \omega_{*} \ll 1$ ), or upon any other linear feature of the drift wave. In this sense, the identification of a locally conserved PV yields a clarification of the technical subtleties surrounding the precise and optimal definition of the adiabatic invariant for use in calculating drift wave - zonal flow interaction.

\section{Discussion and Outlook}

One rather obvious question is whether or not the message of this paper is restricted only to highly simplified models. In particular, its natural to inquire concerning the effects of curvature and the robustness of the theorem in gyrokinetics. Regarding the former, using the approach discussed in this paper, it is straightforward to derive a zonal momentum theorem for electrostatic resistive interchange turbulence[12] in which the pressure evolution equation replaces Eq. (8a), and the interchange drive term is added to Eq. (8b). For this system, the potential vorticity is $u=\omega-p$ and $p$ is the pressure. Proceeding as in Section 3, the momentum balance relation is (for Prandtl number of unity):

$$
\begin{aligned}
\frac{\partial}{\partial t}\left\{\frac{\left\langle\tilde{u}^{2}\right\rangle}{\langle u\rangle^{\prime}}+\left\langle v_{\theta}\right\rangle\right\} & =\left\langle\tilde{v}_{r} \tilde{p}\right\rangle-\frac{1}{\langle u\rangle^{\prime}}\left[D_{0}\left\langle(\nabla \tilde{u})^{2}\right\rangle+\frac{\partial}{\partial r}\left\langle\tilde{v}_{r} \tilde{u}^{2}\right\rangle\right]-\nu\left\langle v_{\theta}\right\rangle \\
& -\frac{\kappa}{\langle u\rangle^{\prime}}\left\langle\left(\nabla^{2} \tilde{v}_{r}\right) \tilde{p}\right\rangle-\frac{\alpha}{\langle u\rangle^{\prime}}\left\langle\tilde{u} \nabla_{\|}^{2} \phi\right\rangle .
\end{aligned}
$$

Here $\alpha$ is a dissipation coefficient. From comparison to Eqn. (11), we see that only the last two terms in Eqn. (18) are new. The first is a curvature-dependent correction to the driving flux, of $O\left(\epsilon k_{\perp}^{2} \rho^{2}\right)$ with respect to $\left\langle\tilde{v}_{r} \tilde{p}\right\rangle$. The second accounts for potential enstrophy damping by resistively modified field line bending (i.e. $\alpha \sim 1 / \eta$ ). Neither substantively alters the messages of the momentum theorem given in Eqn. (11). In particular, while curvature allows a fluctuation-induced torque which breaks local PV 
conservation, its impact on zonal momentum balance is seen to be quite modest. As before, $-\left\langle\tilde{u}^{2}\right\rangle /\langle u\rangle^{\prime}$ emerges as the turbulence pseudomomentum, despite the absence of anything remotely akin to oscillating waves in this application. A discussion of the further extension of this approach to gyrokinetics is beyond the scope of this short paper. We mention in passing, however, that preliminary results indicate such an extension is eminently viable, with the only complications resulting from geodesic sideband couplings to the zonal flow.

More generally, one certainly may ask "What does one gain from all this?", to which we answer:

i.) a momentum balance theorem for zonal flows, which complements existing work on wave-mean flow interaction[13],

ii.) an unambiguous derivation of the pseudomomentum or turbulence momentum density,

iii.) an awareness of the impact of dissipation profiles on determining zonal flow structure,

iv.) an appreciation of the impact of particle transport and turbulence spreading (in particular, potential enstrophy in/out flow) on flow evolution and poloidal spin-up. In particular, a strong link between episodic particle transport events and zonal flow generation is demonstrated. This prediction is easily testable by simulations,

v.) a direct relation between flow momentum evolution and the driving transport flux, which is expressed in terms of macroscopic quantities (such as the driving flux), and is insensitive to details of linear instability, etc. This exact relation may be used in verification of simulations and for the determination of ITB thresholds,

vi.) an approach to understanding zonal flow dynamics in terms of macroscopics and spatial transport, instead of spectral transfer,

vii.) the identification of a new mechanism for saturation of zonal flows in a collisionless plasma.

Many applications of this approach are possible, and will be discussed in future publications.

\section{Acknowledgments}

We thank S.R. Keating, G. Dif-Pradalier, K. Itoh, S.-I. Itoh, C. McDevitt, P.H. Roberts, and A. Soward for many stimulating discussions. This research was supported by U.S. DoE Grant Nos. DE-FG02-04ER-54738 and DE-FG02-08ER54959 (UCSD), U.S. DoE Contract No. DE-AC02-76-CH0-3073 (PPPL), and from NSF Contract No. PHY0551164 (KITP), and benefited from the authors' participation in the 2007 Festival du Theorie, Aix-en-Provence. 


\section{References}

[1] P.H. Diamond, S.-I. Itoh, K. Itoh, and T.S. Hahm, Plasma Phys. Control. Fusion 47, R35, (2005).

[2] K. Itoh, et al., Phys. Plasmas 13, 055502 (2006).

[3] P.B. Rhines and W.R. Holland, Dyn. Atmos. Oceans, 3, 289 (1979).

[4] A. Hasegawa and M. Wakatani, Phys. Rev. Lett. 50, 682 (1983).

[5] J.G. Charney and P.G.Drazin, J. Geophys. Res. 66, 83 (1961).

[6] D.G. Andrews and M.E. McIntyre, J. Fluid Mech. 89, 647 (1978).

[7] G.K. Vallis, Atmospheric and Oceanic Fluid Dynamics, Cambridge University Press (Cambridge), (2006).

[8] A. Hasegawa and K. Mima, Phys. Fluids 21, 87 (1978).

[9] J.R. Myra, D.A. Russell, and D.A. D'lppolito, Phys. Plasmas 15, 032304 (2008).

[10] A. Dimits, et al., Phys. Rev. Lett. 77, 71 (1996).

[11] T.S. Hahm, et al., Plasma Phys. Control. Fusion 46, A323 (2004)

[12] O.D. Gurcan, P.H. Diamond, and T.S. Hahm, Phys. Plasmas 13, 052306 (2006).

[13] P.H. Diamond, et al., Phys. Plasmas 15, 012303 (2008). 